\title{
Anfípodos pelágicos (Amphilochidea, Hyperiidea y Senticaudata) del Pacífico de Costa Rica: nuevos registros y una lista de especies actualizada
}

\section{Pelagic amphipods (Amphilochidea, Hyperiidea and Senticaudata) from the Pacific of Costa Rica: new records and an updated list of species}

\author{
Marco Violante-Huerta ${ }^{1 *}$, Álvaro Morales-Ramírez ${ }^{2}$ \& Laura Sanvicente-Añorve ${ }^{3}$
}

\section{RESUMEN}

En Costa Rica, los estudios sobre zooplancton que aborden los anfípodos pelágicos son escasos, la lista de especies previa a este trabajo incluye solo 41 especies del suborden Hyperiidea. El objetivo de este estudio fue actualizar la lista de especies de anfípodos pelágicos del Pacífico de Costa Rica y registrar especies previamente desconocidas en el país. En julio de 2012, se realizó el muestreo del zooplancton de aguas cercanas a la isla del Coco, Costa Rica, en 26 estaciones oceanográficas. Un total de 13 especies de anfípodos pelágicos de los subórdenes Amphilochidea (1), Hyperiidea (11) y Senticaudata (1) fue identificado para aguas del Pacífico costarricense. Los nuevos registros incrementan el conocimiento de la riqueza de anfípodos pelágicos para el Pacífico de Costa Rica a 54 especies, lo que sugiere la necesidad de explorar más la diversidad de estos organismos en la región.

Palabras clave: isla del Coco, Domo de Costa Rica, Pacífico este tropical, Stenopleura, Synopia

\section{ABSTRACT}

Few studies of zooplankton have been carried out in Costa Rica that address pelagic amphipods; the species list prior to this work includes only 41 species from the suborder Hyperiidea. The aim of this paper was to update the species list of pelagic amphipods from the Pacific of Costa Rica, and record previously unknown species from the country. In July 2012, zooplankton sampling was carried out in the waters near Cocos Island, Costa Rica, at 26 oceanographic stations. A

1 Posgrado en Ciencias del Mar y Limnología, Universidad Nacional Autónoma de México, Av. Ciudad Universitaria 3000, C. P. 04510, Ciudad de México, México; marco_violante@hotmail.com,marco.violante@comunidad.unam.mx* ORCID: https://orcid.org/0000-0002-2199-6711

2 Centro de Investigación en Ciencias del Mar y Limnología y Escuela de Biología, Universidad de Costa Rica, Ciudad de la Investigación, San Pedro, 11501-2060 San José, Costa Rica; alvaro.morales@ucr.ac.cr ORCID: https://orcid.org/0000-0002-0883-5366

3 Instituto de Ciencias del Mar y Limnología, Universidad Nacional Autónoma de México, Circuito Exterior S/N, Ciudad Universitaria, C. P. 04510, Ciudad de México, México; lesa@unam.mx ORCID: https://orcid.org/0000-0002-0951-4564 
total of 13 pelagic amphipod species from the suborders Amphilochidea (1), Hyperiidea (11) and Senticaudata (1) were identified in the Pacific waters of Costa Rica. These new records increase the known total of pelagic amphipod species from the Pacific of Costa Rica to 54 species, which suggests a need to further explore the diversity of these organisms in the region.

Keywords: Cocos Island, Costa Rica Dome, eastern tropical Pacific, Stenopleura, Synopia

\section{INTRODUCCIÓN}

En Costa Rica, son pocos los estudios que abordan la composición, distribución y abundancia de los anfípodos pelágicos presentes en sus aguas. Salmán-Palacios (1985) y Shih y Hendrycks (2003) fueron los primeros en analizar los anfípodos pelágicos de aguas del Pacífico costarricense, contribuyendo al conocimiento de este grupo del zooplancton en la nación. Posteriormente, en un esfuerzo por describir la biodiversidad marina del país, Gasca (2009a) realizó una compilación de los registros históricos de anfípodos hiperídeos del territorio, en la cual enlistó un total de 33 especies del infraorden Physocephalata.

Recientemente, Gasca y Morales-Ramírez (2012) analizaron la composición de los anfípodos hiperídeos alrededor de la isla del Coco, en aguas superficiales de 0-50 $\mathrm{m}$ de profundidad, y registraron 8 especies antes desconocidas para Costa Rica, para sumar un total de 41 especies de hiperídeos en la región costarricense. En su estudio, Gasca y Morales-Ramírez (2012) comentan sobre la necesidad de realizar más exploraciones que traten la diversidad de anfípodos pelágicos de la región, ya que, en zonas cercanas, el número de especies presentes es alrededor de 150 taxones (Gasca, 2009b). Por lo tanto, el objetivo del estudio fue actualizar la lista de especies de anfípodos pelágicos del Pacífico de Costa Rica, incluyendo nuevos registros de distribución de especies de estos organismos, de modo que se contribuya al conocimiento de la riqueza y diversidad de especies del Pacífico este tropical.

\section{MATERIALES Y MÉTODOS}

Las muestras de zooplancton fueron recolectadas durante la campaña oceanográfica UCR-UNA-COCOVI, a bordo del B/I Undersea Hunter, del 7 al 14 de julio de 2012, como parte del proyecto "Interacción océano-atmósfera y la biodiversidad marina del Parque Nacional Isla del Coco, Costa Rica”. El muestreo se realizó en 26 estaciones oceanográficas ordenadas en 3 transectos (160 mn de longitud), en dirección norte-sur, alrededor de la isla del Coco, Costa Rica (Fig. 1; Cuadro 1), por medio de arrastres verticales, con una red de mesozooplancton (49 
cm de boca y $200 \mu \mathrm{m}$ de apertura de malla), entre los 0 y $200 \mathrm{~m}$ de profundidad. El material recolectado se fijó en una solución formalina al $4 \%$.

El material se revisó en laboratorio para la búsqueda de anfípodos; estos fueron separados, preservados en etanol al $70 \%$ y posteriormente se identificaron, utilizando literatura especializada (Stebbing, 1888; Barnard, 1972; Barnard y Thomas, 1989; Vinogradov et al. 1996; Vinogradov, 1999; Hughes, 2009; Zeidler, 1990, 2003, 2004, 2016; Violante-Huerta et al. 2020). El material biológico se encuentra en el Centro de Investigación

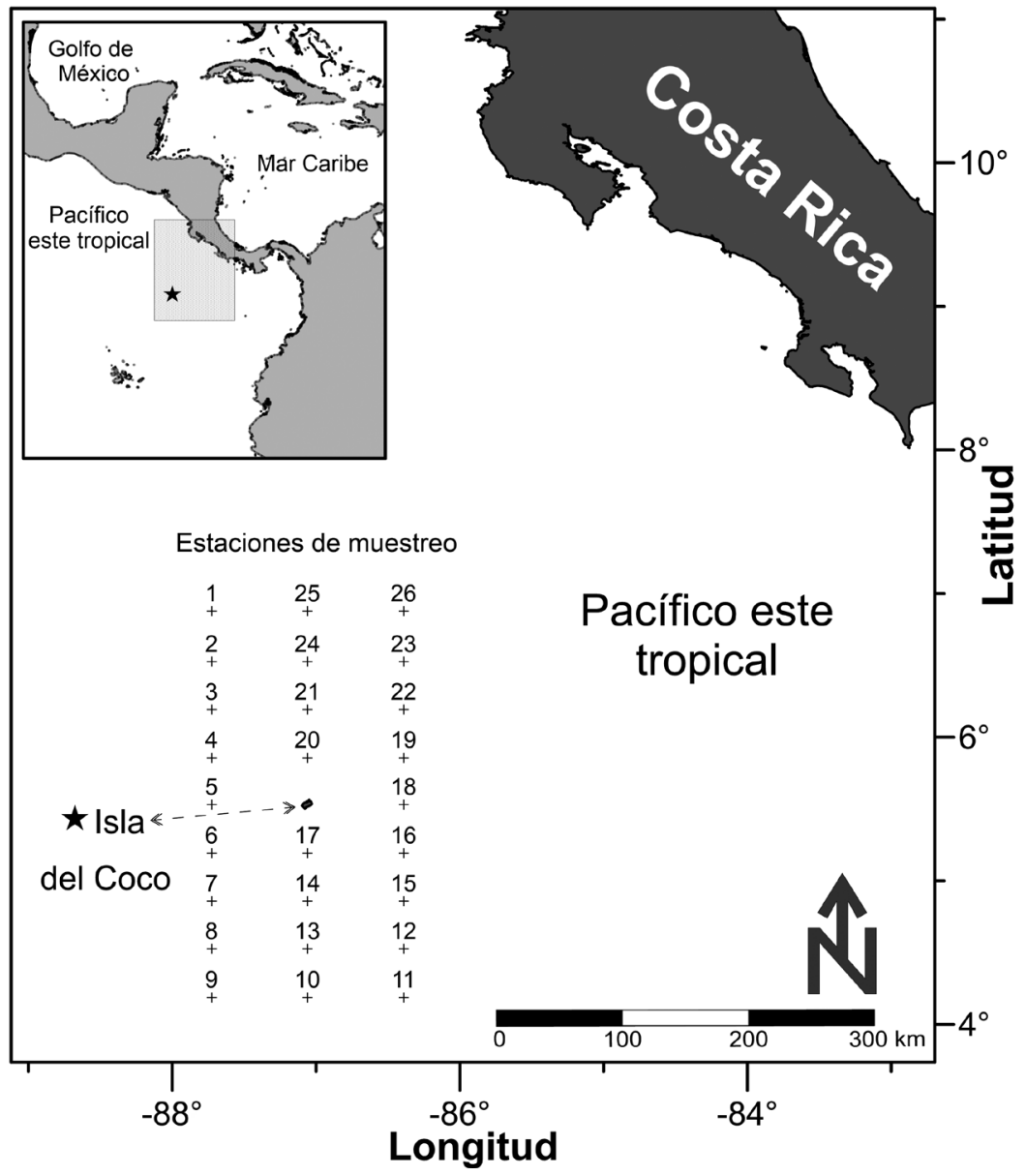

Fig. 1. Localización geográfica de las estaciones de muestreo alrededor del Parque Nacional Isla del Coco, Costa Rica

Fig. 1. Geographical location of the sampling stations around National Park Cocos Island, Costa Rica 
Cuadro 1. Coordenadas geográficas de las estaciones de muestreo en el Pacifico de Costa Rica

Table 1. Geographical coordinates of sampling stations in the Pacific of Costa Rica

\begin{tabular}{clcccc}
\hline Estación & Longitud & Latitud & Estación & Longitud & Latitud \\
\hline $\mathbf{1}$ & -87.725566 & 6.877316 & $\mathbf{1 4}$ & -87.058874 & 4.858456 \\
$\mathbf{2}$ & -87.725566 & 6.525199 & $\mathbf{1 5}$ & -86.389879 & 4.858456 \\
$\mathbf{3}$ & -87.725566 & 6.191748 & $\mathbf{1 6}$ & -86.389879 & 5.192080 \\
$\mathbf{4}$ & -87.725566 & 5.857094 & $\mathbf{1 7}$ & -87.058874 & 5.192080 \\
$\mathbf{5}$ & -87.725566 & 5.530950 & $\mathbf{1 8}$ & -86.389879 & 5.530950 \\
$\mathbf{6}$ & -87.725566 & 5.192080 & $\mathbf{1 9}$ & -86.389879 & 5.857094 \\
$\mathbf{7}$ & -87.725566 & 4.858456 & $\mathbf{2 0}$ & -87.058874 & 5.857094 \\
$\mathbf{8}$ & -87.725566 & 4.525731 & $\mathbf{2 1}$ & -87.058874 & 6.191748 \\
$\mathbf{9}$ & -87.725566 & 4.188336 & $\mathbf{2 2}$ & -86.389879 & 6.191748 \\
$\mathbf{1 0}$ & -87.058874 & 4.188336 & $\mathbf{2 3}$ & -86.389879 & 6.525199 \\
$\mathbf{1 1}$ & -86.389879 & 4.188336 & $\mathbf{2 4}$ & -87.058874 & 6.525199 \\
$\mathbf{1 2}$ & -86.389879 & 4.525731 & $\mathbf{2 5}$ & -87.058874 & 6.877316 \\
$\mathbf{1 3}$ & -87.058874 & 4.525731 & $\mathbf{2 6}$ & -86.389879 & 6.877316 \\
\hline
\end{tabular}

en Ciencias del Mar y Limnología (CIMAR) de la Universidad de Costa Rica y será catalogado por el Museo de Zoología de la Universidad de Costa Rica.

\section{RESULTADOS Y DISCUSIÓN}

Un total de 100 anfípodos pelágicos pertenecientes a 8 géneros y 13 especies fueron observados por primera vez en las aguas oceánicas del $\mathrm{Pa}$ cífico costarricense. Con estos nuevos registros, la lista actualizada de especies de anfípodos pelágicos del territorio mencionado asciende a 54: 1 del suborden Amphilochidea, 52 de Hyperiidea y 1 de Senticaudata (Cuadro 2). 
Cuadro 2. Lista actualizada de los anfípodos pelágicos del Pacífico de Costa Rica, incluye profundidad y registros previos

Table 2. Updated list of the pelagic amphipods from the Pacific of Costa Rica, includes depth and previous records

\begin{tabular}{|c|c|c|c|}
\hline Taxón (referencia ${ }^{a}$ ) & $\begin{array}{l}\text { Profundidad } \\
\text { (m) }\end{array}$ & Taxón (referencia ${ }^{a}$ ) & $\begin{array}{l}\text { Profundidad } \\
\text { (m) }\end{array}$ \\
\hline $\begin{array}{l}\text { Suborden Amphilochidea } \\
\text { Familia Synopiidae } \\
\text { Synopia } \text { sp. }^{6}\end{array}$ & $0-200$ & $\begin{array}{l}\text { Familia Lycaeopsidae } \\
\text { Lycaeopsis themistoides }{ }^{4} \\
\text { Lycaeopsis zamboangae }{ }^{4}\end{array}$ & $\begin{array}{l}0-50 \\
0-50\end{array}$ \\
\hline 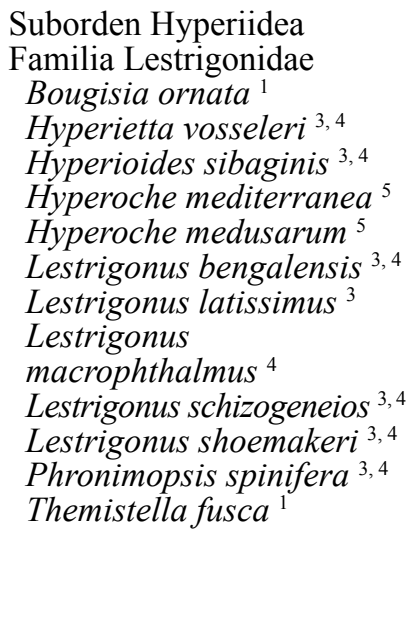 & $\begin{array}{l}0-450 \\
0-50 \\
0-50 \\
\text { superficie } \\
\text { superficie } \\
0-50 \\
\text { superficie } \\
0-50 \\
0-50 \\
0-50 \\
0-50 \\
0-450\end{array}$ & 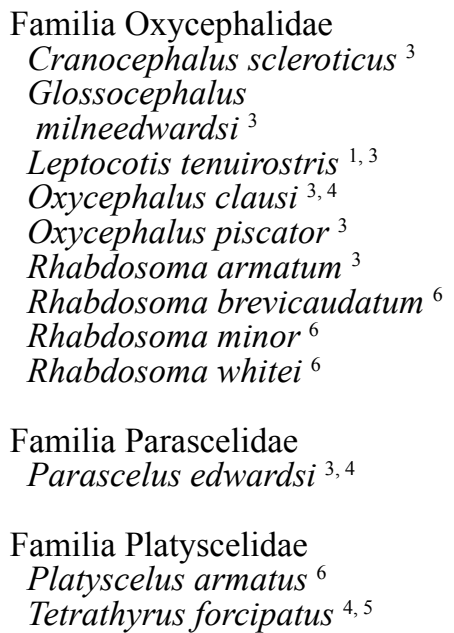 & $\begin{array}{l}\text { superficie } \\
\text { superficie } \\
0-450 \\
0-50 \\
\text { superficie } \\
\text { superficie } \\
0-200 \\
0-200 \\
0-200\end{array}$ \\
\hline $\begin{array}{l}\text { Familia Phronimidae } \\
\text { Phronima bowmani }{ }^{4} \\
\text { Phronima sedentaria }{ }^{1} \\
\text { Phronimella elongata }^{1}\end{array}$ & $\begin{array}{l}0-50 \\
0-450 \\
0-450\end{array}$ & $\begin{array}{l}\text { Familia Paraphronimidae } \\
\text { Paraphronima crassipes } \\
\text { Paraphronima gracilis }^{1,4}\end{array}$ & $\begin{array}{l}0-200 \\
0-450\end{array}$ \\
\hline $\begin{array}{l}\text { Familia Phrosinidae } \\
\text { Anchylomera blossevillei }^{1} \\
\text { Phrosina semilunata }^{1,4} \\
\text { Primno brevidens }^{1,4} \\
\text { Primno latreillei }^{6} \\
\\
\text { Familia Amphithyridae } \\
\text { Amphithyrus sculpturatus }{ }^{3,4}\end{array}$ & $\begin{array}{l}0-450 \\
0-450 \\
0-450 \\
0-200\end{array}$ & $\begin{array}{l}\text { Familia Vibilidae } \\
\text { Vibilia armata }^{2} \\
\text { Vibilia australis }^{2} \\
\text { Vibilia borealis }^{6} \\
\text { Vibilia chuni }^{4} \\
\text { Vibilia cultripes }^{6} \\
\text { Vibilia gibbosa }^{6} \\
\text { Vibilia }^{6} \text { pripes }\end{array}$ & $\begin{array}{l}0-1756 \\
0-1300 \\
0-200 \\
0-50 \\
0-200 \\
0-200 \\
0-250\end{array}$ \\
\hline $\begin{array}{l}\text { Familia Brachyscelidae } \\
\text { Brachyscelus crusculum }{ }^{4} \\
\text { Brachyscelus rapacoides }{ }^{3}\end{array}$ & $\begin{array}{l}0-50 \\
\text { superficie }\end{array}$ & $\begin{array}{l}\text { Familia Scinidae } \\
\text { Scina borealis }{ }^{6} \\
\text { Scina excisa }{ }^{6}\end{array}$ & $\begin{array}{l}0-200 \\
0-200\end{array}$ \\
\hline
\end{tabular}




\begin{tabular}{|c|c|c|c|}
\hline Taxón (referencia ${ }^{a}$ ) & $\begin{array}{l}\text { Profundidad } \\
\text { (m) }\end{array}$ & Taxón (referencia ${ }^{a}$ ) & $\begin{array}{l}\text { Profundidad } \\
\text { (m) }\end{array}$ \\
\hline $\begin{array}{l}\text { Familia Eupronoidae } \\
\text { Eupronoe armata }^{4} \\
\text { Parapronoe parva }^{1,3}\end{array}$ & $\begin{array}{l}0-50 \\
0-450\end{array}$ & $\begin{array}{l}\text { Suborden Senticaudata } \\
\text { Familia Calliopiidae } \\
\text { Stenopleura atlantica }{ }^{6}\end{array}$ & $0-200$ \\
\hline $\begin{array}{l}\text { Familia Lycaeidae } \\
\text { Lycaea pachypoda }^{5} \\
\text { Lycaea vincentii }{ }^{3} \\
\text { Simorhynchotus } \\
\text { antennarius }{ }^{3,4}\end{array}$ & $\begin{array}{l}\text { superficie } \\
\text { superficie } \\
0-50\end{array}$ & & \\
\hline
\end{tabular}

${ }^{a}$ Referencias: ${ }^{1}$ Salmán-Palacios (1985); ${ }^{2}$ Shih y Hendrycks (2003); ${ }^{3}$ Gasca (2009a); ${ }^{4}$ Gasca y

Morales-Ramírez (2012); ${ }^{5}$ Gasca (com. pers. junio, 2019); ${ }^{6}$ este estudio.

Orden AMPHIPODA Latreille, 1816 Suborden AMPHILOCHIDEA Boeck, 1871

Familia SYNOPIIDAE Dana, 1853 Género Synopia Dana, 1852 Synopia sp.

(Fig. 2A)

Diagnóstico. Cabeza con rostro protuberante y redondeado, ojos y ojos accesorios presentes. Segmentos 1-2 de la antena 1 simples. Gnatópodos 1-2 ligeramente subquelados, simples y pequeños. Pleonitos 1-3 con márgenes lisos. Urópodo 3 más largo que los urópodos 1-2. Telson pequeño con una hendidura. Fuentes: Barnard (1972), Barnard y Thomas (1989), Hughes (2009).

Material examinado. 4 individuos: 1 ind, est $6 ; 1$ ind, est $7 ; 1$ ind, est $11 ; 1$ ind, est 17 .

Distribución global. Atlántico norte: isla Guadalupe, Venezuela, Belice, Caribe mexicano, golfo de México, Bermudas, EE. UU. Atlántico sur: Brasil, golfo de Guinea. Pacífico norte: Japón, Alaska, Kiribati. Pacífico sur: Australia, Papúa Nueva Guinea, Nueva Caledonia, Polinesia Francesa, Ecuador. Océano Índico: Madagascar, mar Rojo, Indonesia, Australia (Shoemaker, 1945; Ledoyer, 1986; Hughes, 2009; GBIF.org, 2020; OBIS, 2020; Senna et al. 2020).

Discusión taxonómica. El material observado coincide con las características diagnósticas del género Synopia, sin embargo, la determinación en el nivel de especie no fue realizada en el presente trabajo, por lo que se considera solo el registro del género, por primera vez, para el Pacífico costarricense. Este género es reconocido por mostrar gran variabilidad morfológica, principalmente en la especie tipo $S$. ultramarina (Shoemaker, 1945; Ledoyer, 1986; Barnard y Thomas, 1989; Hughes, 2009).

\section{Comentarios generales. El} género Synopia ha sido registrado mayormente en aguas de la zona epipelágica de los océanos $(0-200 \mathrm{~m}$ de profundidad) o en asociación con suelos blandos y su distribución es circumtropical (Shoemaker, 1945; 
Barnard, 1972; Vinogradov, 1999; Senna et al. 2020). En el Pacífico este tropical, se han registrado 3 especies del género: $S$. angustiformes, $S$. scheeleana y, aparentemente, S. ultramarina (Barnard, 1972; Barnard y Karaman, 1991; GBIF.org, 2020).
Suborden HYPERIIDEA H. Milne

Edwards, 1830

Familia PHROSINIDAE Dana, 1852

Género Primno Guérin-Méneville, 1836

Primno latreillei Stebbing, 1888

(Fig. 2B)

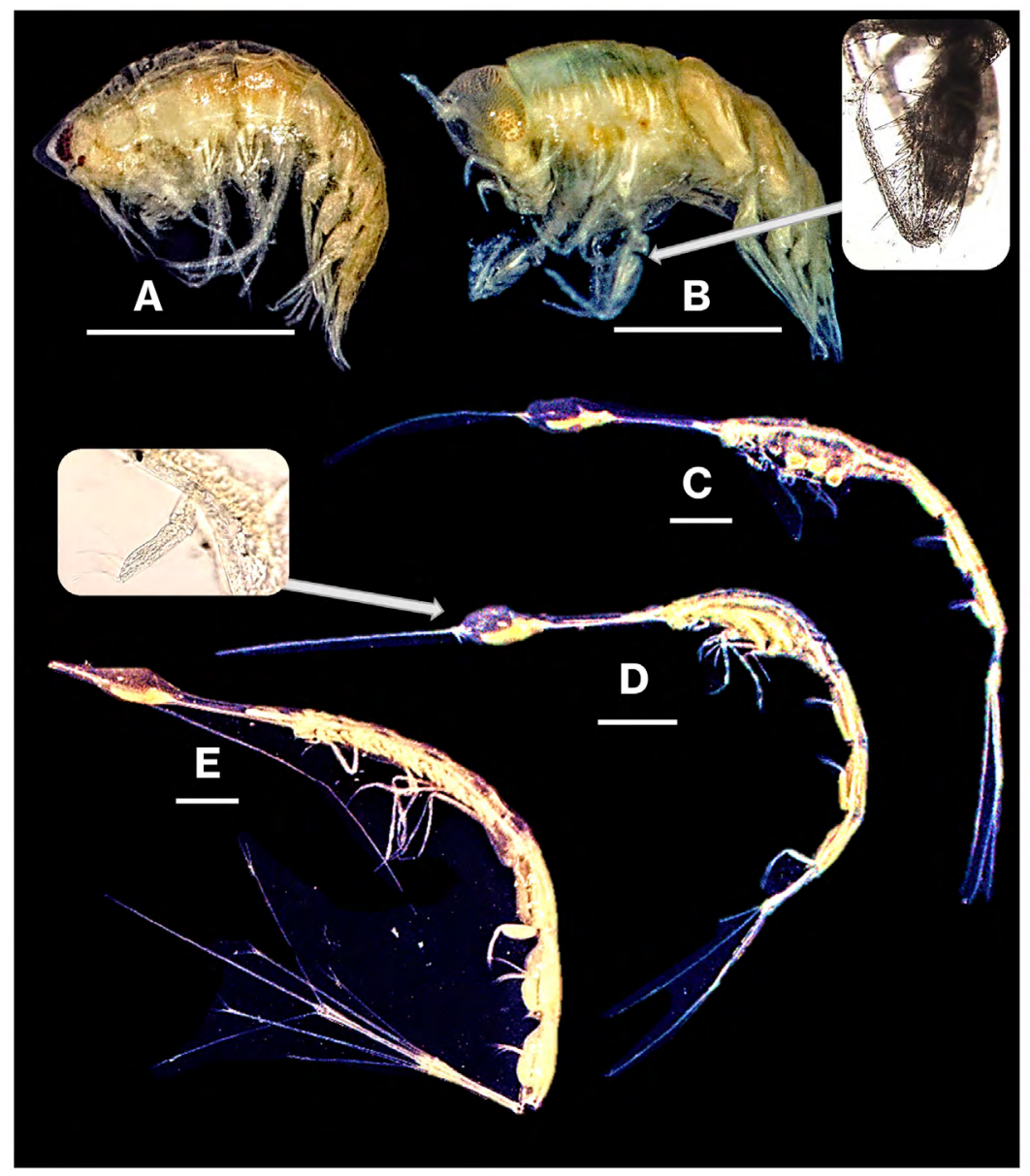

Fig. 2. Nuevos registros de anfípodos pelágicos del Pacífico de Costa Rica. A, Synopia sp.; B, Primno latreillei, que muestra el pereiópodo 5; C, Rhabdosoma brevicaudatum; $\mathrm{D}, R$. minor, que presenta la antena 1 dividida en 2 segmentos; $\mathrm{E}, R$. whitei. Barras de escala: $0.5 \mathrm{~mm}$

Fig. 2. New records of pelagic amphipods from the Pacific of Costa Rica. A, Synopia sp.; B, Primno latreillei, showing the pereopod 5; C, Rhabdosoma brevicaudatum; D, $R$. minor, presenting the antenna 1 divided in 2 segments; E, $R$. whitei. Scale bars: $0.5 \mathrm{~mm}$ 
Diagnóstico. Cuerpo robusto, especialmente el pereión. Pereiópodo 5 con dientes largos en el margen anterior del carpo, la longitud de estos similar al ancho del carpo. Adicionalmente, el margen proximal anterior del carpo del pereiópodo 5 con un diente largo. Pereiópodo 7 con el basis $1.5 \mathrm{x}$ más largo que la longitud de los demás segmentos combinados. Fuentes: Vinogradov et al. (1996), Vinogradov (1999), Zeidler (2004).

Material examinado. 33 individuos: 1 ind, est 4; 12 ind, est 6; 3 ind, est 7; 3 ind, est $10 ; 1$ ind, est $11 ; 2$ ind, est $12 ; 1$ ind, est $15 ; 1$ ind, est $16 ; 1$ ind, est 17; 3 ind, est 18; 1 ind, est 19; 2 ind, est 20; 2 ind, est 21.

Distribución global. Atlántico norte: Guyana, Caribe mexicano, golfo de México, mar de Sargazo, EE. UU., mar Mediterráneo. Atlántico sur: golfo de Guinea. Pacífico norte: China, giro central de Pacífico norte, México, Colombia. Pacífico sur: Australia, mar de Tasmania, Perú. Océano Índico: mar Rojo (Shulenberger, 1978; Vinogradov, 1991, 1999; Vinogradov et al. 1996; Zeidler, 2004; Gasca, 2009b, 2009c; Valencia et al. 2013; Zhang et al. 2014; GBIF.org, 2020; OBIS, 2020).

Discusión taxonómica. Esta especie se distingue del resto de las del género Primno por presentar dientes largos (tan largos como el ancho del campo) en el margen anterior del carpo del pereiópodo 5; además, tiene un diente largo proximal. Algunos expertos sugieren que $P$. latreillei es una especie sinónima de $P$. johnsoni, debido a que la diferencia de la longitud del basis del pereiópodo 7 podría deberse al desarrollo ontogénico de la especie (Zeidler, 2004).

Comentarios generales. Esta especie fue la más abundante y frecuente en el área de estudio, similar a lo registrado por Valencia et al. (2013) para aguas de Colombia. Este nuevo registro representa una ampliación del rango de distribución geográfica para $P$. latreillei de aprox. $800 \mathrm{~km}$, considerando el registro más cercano por Valencia et al. (2013) en Colombia. El intervalo de distribución vertical registrada para $P$. latreillei es muy amplio, entre 0 y 1000 $\mathrm{m}$ de profundidad (Vinogradov, 1999).

\section{Familia OXYCEPHALIDAE Bate,} 1862

Género Rhabdosoma White, 1847

Rhabdosoma brevicaudatum Stebbing, 1888

(Fig. 2C)

Diagnóstico. Cuerpo extremadamente delgado y largo, con el rostro muy largo, puntiagudo y frágil. Antena 1 consiste en un segmento basal, el callinóforo y un segmento adicional pequeño. Pleón más largo que el pereión. Telson con la punta redondeada, su longitud no alcanza el limité del urópodo 2. Fuentes: Vinogradov et al. (1996), Vinogradov (1999), Zeidler (2016).

Material examinado. 1 individuo, est 6. 
Distribución global. Atlántico norte: Venezuela, mar de Sargazo, Bermudas, mar Mediterráneo, islas Canarias, Guinea-Bissau. Atlántico sur: Sudáfrica. Pacífico norte: Japón, México, Colombia. Pacífico sur: Australia, Perú. Océano Índico: este de Australia (Thurston, 1976a; Vinogradov, 1991, 1999; Vinogradov et al. 1996; Valencia et al. 2013; GBIF.org, 2020; OBIS, 2020).

Discusión taxonómica. Esta especie se distingue de $R$. armatum y $R$. whitei porque presenta la punta del telson redondeada y su longitud no alcanza la del urópodo 2 (Zeidler, 2016). La longitud del telson en $R$. brevicaudatum tiene variaciones que podrían confundirse con $R$. minor; esto ha hecho sospechar sobre la posible sinonimia entre ambas especies (Vinogradov et al. 1996).

Comentarios generales. Rhabdosoma brevicaudatum prefiere habitar aguas superficiales entre 0 y 50 de profundidad (Thurston, 1976a; Vinogradov, 1999). La distribución geográfica de $R$. brevicaudatum se amplía aprox. $800 \mathrm{~km}$ en el Pacífico este, considerando el registro más cercano en Colombia (Valencia et al. 2013).

Rhabdosoma minor Fage, 1954 (Fig. 2D)

Diagnóstico. Cuerpo extremadamente delgado y largo, con el rostro muy largo y puntiagudo. Antena 1 dividida en 2 segmentos: la base y el callinóforo. Pleón más largo que el pereión. Telson con el margen distal redondo, que alcanza la punta del urópodo 2. Fuentes: Vinogradov et al. (1996), Zeidler (2016).

Material examinado. 8 individuos: 1 ind, est 3; 6 ind, est $6 ; 1$ ind, est 12 .

Distribución global. Atlántico norte: Caribe mexicano, mar de Labrador, giro tropical del Atlántico norte entre $12-17^{\circ} \mathrm{N}$ y $32-36^{\circ} \mathrm{W}$, cerca de islas Canarias, golfo de Guinea. Atlántico sur: giro del Atlántico sur entre $11-30^{\circ} \mathrm{S}$ y $25-27^{\circ} \mathrm{W}$, Angola, Sudáfrica. Pacífico norte: China, $0.15^{\circ} \mathrm{N}$ y $106.66^{\circ} \mathrm{W}$, México, Colombia. Pacífico sur: islas Salomón, Perú. Océano Índico: Madagascar, Mozambique (Dick, 1967; Vinogradov, 1991; Gasca, 2009c; Valencia et al. 2013; Zhang et al. 2014; Burridge et al. 2017; GBIF. org, 2020; OBIS, 2020).

\section{Discusión taxonómica. Esta} especie es muy similar a $R$. brevicaudatum por la forma redondeada de la punta del telson, pero se distinguen principalmente porque en $R$. minor la longitud del telson sí alcanza la del urópodo 2 (Zeidler, 2016), a diferencia de $R$. brevicaudatum, en el cual la longitud del telson es claramente menor a la del urópodo 2. Además, la antena 1 de $R$. minor únicamente está dividida en 2 segmentos (base y callinóforo), mientras que $R$. brevicaudatum tiene 3 .

Comentarios generales. La distribución vertical de esta especie alcanza los $300 \mathrm{~m}$ de profundidad y la 
geográfica es circumtropical (Vinogradov, 1999). Rhabdosoma minor presentó una amplitud en su distribución de aprox. $800 \mathrm{~km}$ en el Pacífico este, ya que el registro más cercano fue en Colombia (Valencia et al. 2013).

Rhabdosoma whitei Bate, 1862 (Fig. 2E)

Diagnóstico. Cuerpo extremadamente delgado y largo, con el rostro muy largo y puntiagudo. Antena 1 dividida en 2 segmentos: la base y el callinóforo. Pereiópodo 1 con el proceso carpal simple, sin dientes. Pleón ligeramente más largo que el pereión. Urópodos 2-3 con los exópodos bien desarrollados. Telson con el margen distal puntiagudo, más largo que el urópodo 2. Fuentes: Vinogradov et al. (1996), Zeidler (2016).

Material examinado. 1 individuo, est 15.

Distribución global. Atlántico norte: Venezuela, Caribe mexicano, golfo de México, EE. UU., islas Canarias, golfo de Guinea (Senegal-Gabón). Atlántico sur: Uruguay, Brasil, Sudáfrica, aguas entre Sudamérica y África en $8.2^{\circ} \mathrm{S}$ y $18^{\circ} \mathrm{W}$. Pacífico norte: China, México, Colombia. Pacífico sur: Australia, Perú. Océano Índico: Sudáfrica, Mozambique, Madagascar, Indonesia (Dick, 1967; Vinogradov, 1991; Gasca, 2009b, 2009c; Valencia et al. 2013; Zhang et al. 2014; GBIF. org, 2020; OBIS, 2020).

\section{Discusión}

taxonómica.

Esta especie es la más cercana morfológicamente a $R$. armatum, previamente registrada en aguas costarricenses; ambas presentan la punta del telson aguda y su longitud es mayor a la del urópodo 2, sin embargo, en $R$. whitei los exópodos de los urópodos 2-3 están bien desarrollados, mientras que en $R$. armatum son muy pequeños (Zeidler, 2016).

Comentarios generales. La especie prefiere habitar en aguas epipelágicas (Vinogradov, 1999). Por otro lado, Vinogradov et al. (1996) sugieren que $R$. whitei también podría habitar aguas profundas por debajo de los $200 \mathrm{~m}$ de profundidad. El registro geográfico más cercano a la isla del Coco se localizó a aprox. $800 \mathrm{~km}$ al este, en Colombia, donde $R$. whitei mostró baja abundancia y frecuencia (Valencia et al. 2013), similar a lo observado en este estudio.

Familia PLATYSCELIDAE Bate, 1862

Género Platyscelus Spence Bate, 1861 Platyscelus armatus (Claus, 1879)

(Fig. 3A)

Diagnóstico. Cuerpo ancho $\mathrm{y}$ redondeado, con todos los somitas del pereión y pleón convexos. El propodio del gnatópodo 1 con el margen anterior liso. Coxa del pereiópodo 5 con un proceso espiniforme largo proyectado lateralmente. Coxas 5-6 más largas que el resto. Segmento basal del pereiópodo 6 con una fisura pequeña, tan larga como la mitad de la longitud del mero. Fuentes: Vinogradov et al. 
(1996), Vinogradov (1999), Zeidler (2016).

Material examinado. 23 individuos: 7 ind, est $6 ; 2$ ind, est $8 ; 3$ ind, est $10 ; 7$ ind, est $11 ; 3$ ind, est $12 ; 1$ ind, est 13.

Distribución global. Atlántico norte: Venezuela, golfo de México, al norte de las islas Azores, golfo de Guinea-Liberia. Atlántico sur: Brasil, Angola, Namibia, Sudáfrica. Pacífico norte: Hawái, golfo de California, México. Pacífico sur: Australia, Nueva Caledonia, Nueva Zelanda, Indonesia-Estrecho de Macasar, Perú. Océano Índico: Tanzania (Vinogradov, 1991; Vinogradov et al. 1996; Violante-Huerta, 2019; GBIF.org, 2020; OBIS, 2020).

Discusión taxonómica. Platyscelus armatus es fácilmente identificada por la presencia de un proceso espiniforme en la coxa del pereiópodo 5 (Zeidler, 2016). En vista dorsal, la especie muestra un par de espinas laterales que no se encuentran en el resto de las especies del género Platyscelus.

Comentarios generales. Platyscelus armatus ha sido registrada mayormente en aguas superficiales de los océanos, pero también se encuentra en las de la zona mesopelágica hasta los $800 \mathrm{~m}$ de profundidad (Vinogradov et al. 1996; Vinogradov, 1999). En el Pacífico este, la distribución geográfica de $P$. armatus se extiende al norte de México y al sur de Perú (Vinogradov, 1991; GBIF.org, 2020), lo que representa una amplitud en su rango de distribución de más de $2600 \mathrm{~km}$ hacia el sur y al norte.

\section{Familia PARAPHRONIMIDAE}

Bovallius, 1887

Género Paraphronima Claus, 1879

Paraphronima crassipes Claus, 1879

(Fig. 3B)

Diagnóstico. Cabeza grande con forma de cubo, con los ojos cubriendo gran cantidad de su superficie. Pereiópodo 7 ligeramente más corto que el anterior. Margen ventral de la placa epimeral del pleonito 1 claramente redondo y perpendicular al eje del cuerpo. Fuentes: Vinogradov et al. (1996), Zeidler (2003).

Material examinado. 6 individuos: 1 ind, est 4; 2 ind, est $6 ; 3$ ind, est 7 .

Distribución global. Atlántico norte: Venezuela, Caribe mexicano, golfo de México, EE. UU., Islas Turcas y Calcos, Bermudas, aguas entre $23.5-36.1^{\circ} \mathrm{N}$ y $26-39.46^{\circ} \mathrm{W}$, mar Mediterráneo, Guinea. Atlántico sur: $2.33^{\circ} \mathrm{S}$ y $12.75^{\circ} \mathrm{W}$, Angola, Namibia, Sudáfrica. Pacífico norte: Japón, mar de Bering, EE. UU., México, Colombia. Pacífico sur: Nueva Zelanda, Australia, Perú. Océano Índico: Sudáfrica, Mozambique, Madagascar, $12.07^{\circ} \mathrm{S}$ y $70.03^{\circ} \mathrm{E}$, zona sur entre $41.8-45.88^{\circ}$ S y $84.55-110^{\circ} \mathrm{E}$ (Vinogradov, 1991; Vinogradov et al. 1996; Gasca, 2009b, 2009c; Valencia et al. 2013; GBIF.org, 2020; OBIS, 2020). 


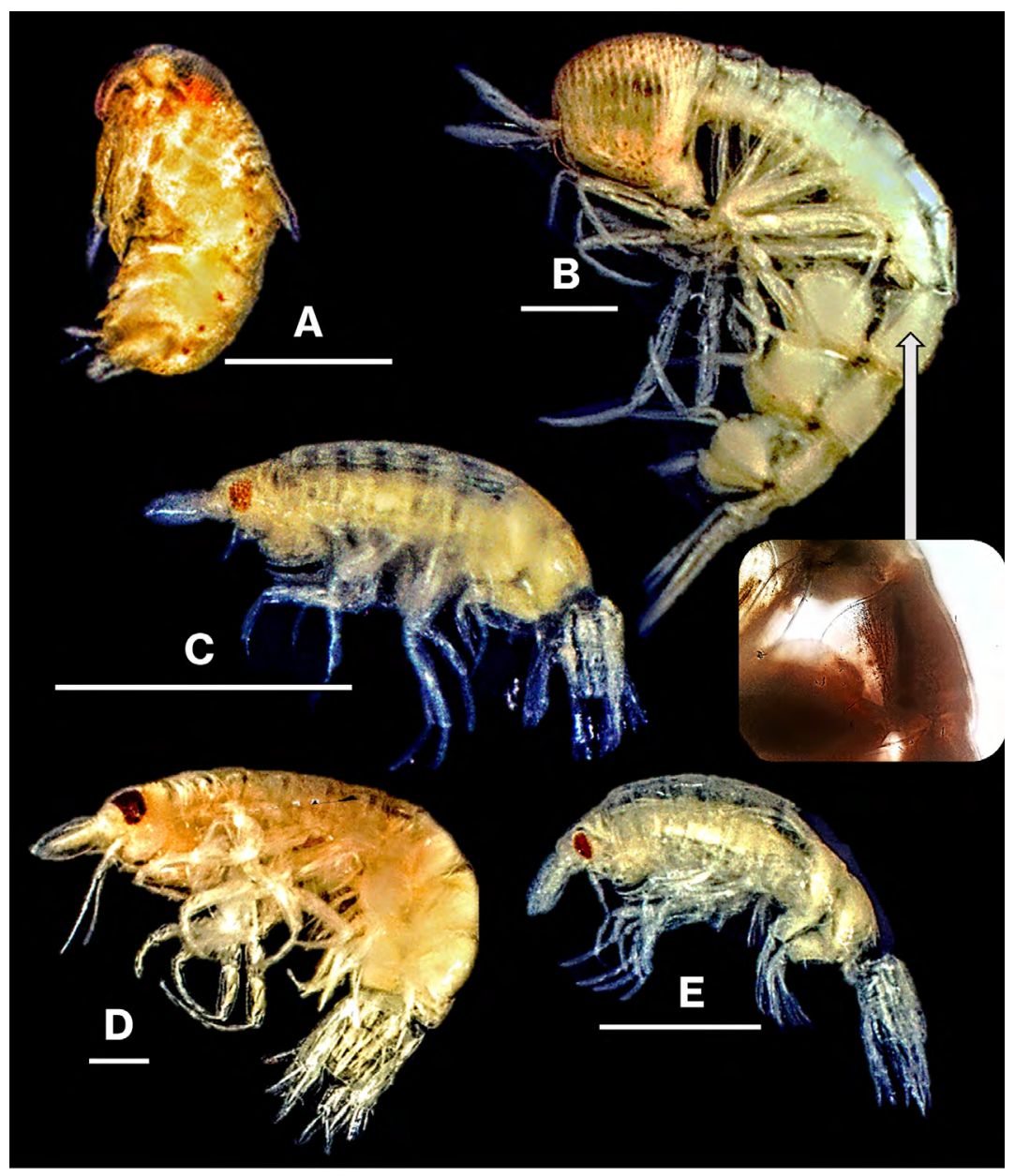

Fig. 3. Nuevos registros de anfípodos pelágicos del Pacífico de Costa Rica. A, Platyscelus armatus; B, Paraphronima crassipes, que muestra la placa epimeral 1 redonda y perpendicular al eje del cuerpo; C, Vibilia borealis; D, V. cultripes; E, V. gibbosa. Barras de escala: $0.5 \mathrm{~mm}$

Fig. 3. New records of pelagic amphipods from the Pacific of Costa Rica. A, Platyscelus armatus; B, Paraphronima crassipes, showing the epimeral plate 1, rounded and perpendicular to body axis; C, Vibilia borealis; D, V. cultripes; E, V. gibbosa. Scale bars: $0.5 \mathrm{~mm}$

Discusión taxonómica. Esta somita tiene forma redondeada y está especie se distingue de $P$. gracilis direccionada perpendicular al eje del por la forma del margen inferior de cuerpo, mientras que para P. gracilis, la placa epimeral del primer pleoni- la placa epimeral forma un ángulo to, principalmente. En P. crassipes, el agudo con respecto al eje del cuerpo 
(Zeidler, 2003). Además, el preiópodo 6 es mucho más largo que el 7 en $P$. gracilis, a diferencia de $P$. crassipes, en el cual ambos pereiópodos son más cercanos en longitud (Zeidler, 2003), como se observó en los ejemplares de este estudio.

Comentarios generales. La especie se ha reportado en aguas epi- y mesopelágicas hasta los $720 \mathrm{~m}$ de profundidad (Vinogradov, 1999), con mayor frecuencia y abundancia durante el día en aguas superficiales (Thurston, 1976a). El registro geográfico más cercano en el Pacífico este para P. crassipes fue en Colombia (Valencia et al. 2013), lo que representa una amplitud del rango de distribución de aprox. 800 km en el Pacífico este.

Familia VIBILIIDAE Dana, 1852

Género Vibilia H. Milne Edwards, 1830

Vibilia borealis Bate \& Westwood, 1868

(Fig. 3C)

Diagnóstico. Cuerpo robusto. Ojos ovalados. Antena 1 con el flagelo ligeramente más ancho que el pedúnculo, el margen distal redondo. Dáctilo del pereiópodo 3-4 delgado, más de $0.2 \mathrm{x}$ del largo del propodio. Pereiópodo 7 con la base más corta que la longitud del resto de los segmentos combinados. Márgenes postero-laterales del último urosomita más o menos iguales que el margen distal (sin proyecciones). Urópodo 2 más corto que el urópodo 3. Telson redondo. Fuentes: Vinogradov et al. (1996), Zeidler (2003).

Material examinado. 2 individuos: 1 ind, est 12; 1 ind, est 14.

Distribución global. Atlántico norte: México, Irlanda, Noruega, España, Portugal, Marruecos. Atlántico sur: $4.0^{\circ} \mathrm{S}$ y $8.25^{\circ}$ E. Pacífico norte: México, entre $33.13-33.37^{\circ} \mathrm{N}$ y 118.17-118.52 ${ }^{\circ}$ W. Pacífico sur: Nueva Zelanda, islas Molucas, mar de Sulú, Perú (Vinogradov, 1991; Vinogradov et al. 1996; Shih \& Hendrycks, 2003; Zeidler, 2003; Gasca, 2009b; GBIF. org, 2020; OBIS, 2020).

Discusión taxonómica. Esta especie se asemeja más a $V$. jeangerardi por el dáctilo corto de los pereiópodos 5-6 (0.2x la longitud del propodio), sin embargo, $V$. borealis tiene la primera antena más grande, el dáctilo de los pereiópodos 3-4 más largo y delgado, y el telson más triangular que $V$. jeangeardi (Zeidler, 2003).

Comentarios generales. Esta especie prefiere habitar en aguas frías, pero también se ha recolectado en zonas tropicales de los océanos Atlántico y Pacífico. Particularmente, en el Pacífico este, $V$. borealis fue registrada al norte de México (Gasca, 2009b) y al sur de Perú (Vinogradov, 1991), lo que representa una amplitud en su distribución geográfica de aprox. $2600 \mathrm{~km}$ al sur y al norte del Pacífico americano. 
Vibilia cultripes Vosseler, 1901 (Fig. 3D)

Diagnóstico. Cuerpo ancho. Ojos ovalados. El flagelo de la antena 1 es ovalado y con el margen distal redondo. Pereiópodo 7 con procesos bulbosos en el margen anterodistal del carpo y propodio, dáctilo lanceolado. Márgenes postero-laterales del último urosomita proyectados junto al pedúnculo del urópodo 3 , ligeramente más largos que el margen distal. Urópodo 3 con el pedúnculo tan lago como el endópodo. Telson redondo. Fuentes: Vinogradov et al. (1996), Zeidler (2003).

Material examinado. 1 individuo, est 25.

Distribución global. Atlántico norte: Bermudas, EE. UU., islas Azores, España, Portugal, islas Canarias, mar Mediterráneo, Cabo Verde, Sierra Leona. Atlántico sur: Guinea Ecuatorial, Namibia, $13.0^{\circ} \mathrm{S}$ y $21.6^{\circ} \mathrm{W}, 35.23^{\circ} \mathrm{S}$ y $6.81^{\circ} \mathrm{E}$. Pacífico norte: mar de Japón, EE. UU., golfo de California, México, entre $11-34^{\circ} \mathrm{N}$ y $107-119^{\circ}$ W. Pacífico sur: Australia, Perú. Océano Índico ecuatorial (Vinogradov, 1991, 1999; Vinogradov et al. 1996; Shih \& Hendrycks, 2003; Zeidler, 2003; Gasca, 2009b; GBIF. org, 2020; OBIS, 2020).

Discusión taxonómica. Esta especie se asemeja morfológicamente a $V$. longicarpus, no obstante, en $V$. cultripes, el endópodo del urópodo 3 tiene 2 dientes terminales, ausentes en $V$. longicarpus (Zeidler, 2003). Además, en $V$. cultripes se distinguen claramente los procesos bulbosos grandes del margen anterodistal del carpo y propodio del pereiópodo 7 , mientras que en $V$. longicarpus los procesos son muy pequeños (Zeidler, 2003).

Comentarios generales. Vibilia cultripes prefiere habitar aguas entre 0 y $300 \mathrm{~m}$ de profundidad, presente en baja abundancia (Vinogradov, 1999, Zeidler, 2003). La distribución de $V$. cultripes en el Pacífico este se amplía aprox. $2600 \mathrm{~km}$ desde el sur de Perú (Vinogradov, 1991) y desde el norte de México (Gasca, 2009b).

Vibilia gibbosa Bovallius, 1887

(Fig. 3E)

Diagnóstico. Cuerpo robusto. Ojos ovalados. Antena 1 tan larga como el primer somita del pereión, con el flagelo más ancho que el pedúnculo y el margen distal redondeado. Pereiópodos 5-6 con el dáctilo más largo que $0.3 \mathrm{x}$ de la longitud del propodio. Pereiópodo 7 con la base más corta que la longitud del resto de los segmentos combinados. Márgenes postero-laterales del último urosomita más o menos iguales que el margen distal (sin proyecciones). Urópodo 2 más corto que el urópodo 3. Telson redondo. Fuentes: Vinogradov et al. (1996), Zeidler (2003).

Material examinado. 2 individuos: 1 ind, est 12; 1 ind, est 20 .

Distribución global. Atlántico norte: Caribe mexicano, golfo de México, islas Turcas y Caicos, mar Mediterráneo. Atlántico sur: 0-20 $\mathrm{S}$. Pacífico 
norte: China, Japón, EE. UU., México. Pacífico sur: Perú (Vinogradov, 1991, 1999; Vinogradov et al. 1996; Zeidler, 2003; Gasca, 2009b; Zhang et al. 2014; GBIF.org, 2020; OBIS, 2020).

\section{Discusión taxonómica. Esta} especie es fácilmente diferenciada del resto de las del género Vibilia por el flagelo bulboso de la antena 1 (Zeidler, 2003). Entre las especies más similares del género, $V$. propinqua es la más cercana, ya que comparte muchas de las características, sin embargo, se distinguen fácilmente por la forma del telson, punteado en $V$. propinqua y redondeado en $V$. gibbosa (Zeidler, 2003).

Comentarios generales. Esta especie habita la zona epipelágica y mesopelágica, registrada por encima de $500 \mathrm{~m}$ de profundidad (Vinogradov, 1999). El registro geográfico más cercano en el Pacífico este tropical, para V. gibbosa, fue al norte de México (Gasca, 2009b) y al sur de Perú (Vinogradov, 1991), lo que representa una amplitud en su distribución geográfica de aprox. $2600 \mathrm{~km}$ al sur y al norte del Pacífico americano.

Familia SCINIDAE Stebbing, 1888

Género Scina Prestandrea, 1833

Scina borealis (G. O. Sars, 1882) (Fig. 4A)

Diagnóstico. Cuerpo delgado con su superficie lisa y sin quillas. Antena 1 delgada ligeramente más larga que el pereión. Pereiópodo 5, base con los márgenes anterior y posterior aserrados y con el margen distal proyectado sobre el isquio, mero tan largo como el carpo, propodio más corto que el mero. Urópodos 1-2 con los exopoditos reducidos a pequeñas espinas. Urópodo 1 armado de espinas largas en el margen interno. Urópodo 2 con el margen anterior liso y el margen posterior denticulado. Telson triangular y pequeño. Fuentes: Zeidler (1990), Vinogradov et al. (1996).

Material examinado. 2 individuos: 1 ind, est 20; 1 ind, est 22.

Distribución global. Presente en todos los océanos del mundo en aguas tropicales, templadas y frías, desde el Ártico $\left(80^{\circ} \mathrm{N}\right)$ hasta el Antártico $\left(72^{\circ}\right.$ S). En el Pacífico este, se ha registrado en EE. UU., México, Colombia, Ecuador, Perú y Chile (Vinogradov, 1991; Vinogradov et al. 1996; Gasca, 2009b; Valencia et al. 2013; GBIF.org, 2020; OBIS, 2020).

Discusión taxonómica. Esta especie se asemeja más morfológicamente a $S$. wagleri, por presentar el margen interno del urópodo 1 aserrado (con espinas largas), pero se diferencia porque en $S$. borealis los urópodos son más anchos y los exopoditos de los urópodos 1-2 están reducidos a espinas pequeñas (Vinogradov et al. 1996).

Comentarios generales. En el plano vertical, $S$. borealis tiene una distribución muy amplia que va de $\operatorname{los} 50$ a los $3000 \mathrm{~m}$ de profundidad, presente en aguas frías, templadas y tropicales (Vinogradov et al. 1996; 
Vinogradov, 1999). Además, S. borealis es reconocida como la especie más abundante del género Scina en todos los océanos (Zeidler, 1990; Vinogradov et al. 1996). Su registro geográfico más cercano al área de estudio en el Pacífico este fue a aprox. $800 \mathrm{~km}$ de distancia en aguas de Colombia, donde se observó en baja abundancia (Valencia et al. 2013), similar a lo observado cerca de la isla del Coco.
Scina excisa Wagler, 1926

(Fig. 4B)

Diagnóstico. Cuerpo delgado con su superficie lisa y sin quillas. Antena 1 ligeramente más corta que el pereión. Pereiópodo 5, base con el margen anterior aserrado y con el margen distal proyectado sobre el isquio, mero igual o ligeramente más corto que el carpo. Urópodos 1-2 con los exopoditos reducidos a pequeñas

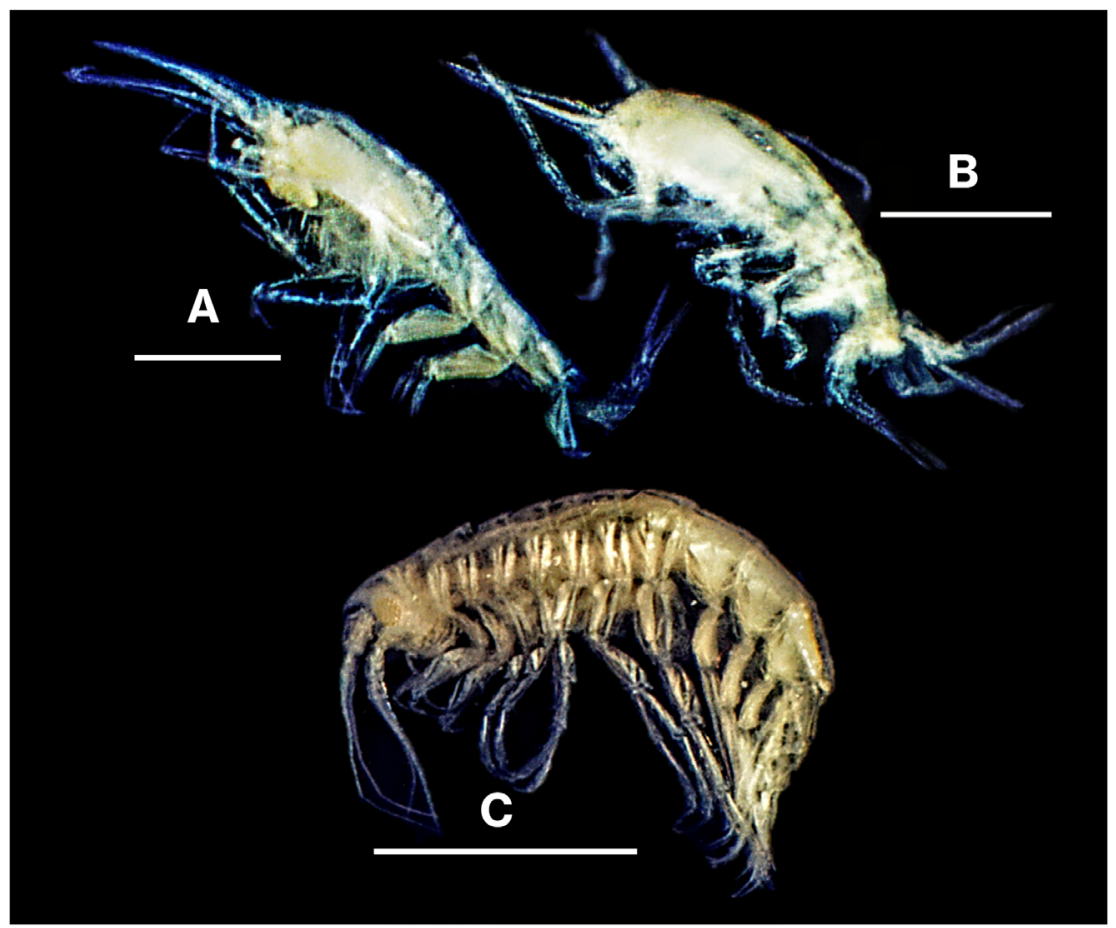

Fig. 4. Nuevos registros de anfípodos pelágicos del Pacífico de Costa Rica. A, Scina borealis; B, S. excisa; C, Stenopleura atlantica. Barras de escala: $0.5 \mathrm{~mm}$

Fig. 4. New records of pelagic amphipods from the Pacific of Costa Rica. A, Scina borealis; B, S. excisa; C, Stenopleura atlantica. Scale bars: $0.5 \mathrm{~mm}$ 
espinas. Urópodo 1 con todo el margen interno liso. Urópodo 2 con el margen anterior ensanchado, curvado bruscamente por encima del nivel de la base del exópodito. Telson triangular con la punta aguda. Fuentes: Zeidler (1990), Vinogradov et al. (1996).

Material examinado. 1 individuo, est 17.

Distribución global. Atlántico norte: Caribe mexicano, golfo de México, Bahamas, mar del Sargazo, islas Canarias. Atlántico sur: Sudáfrica. Pacífico norte: México. Pacífico sur: $4.95^{\circ} \mathrm{S}$ y $119^{\circ} \mathrm{W}$, Perú. Antártica (Vinogradov, 1991; Gasca, 2009b, 2009c; Valencia et al. 2013; Violante-Huerta, 2019; GBIF.org, 2020; OBIS, 2020).

Discusión taxonómica. Esta especie es muy similar a $S$. damasi por la forma de los pereiópodos, pero difiere porque en $S$. excisa el margen interno del urópodo 2 se encorva bruscamente a la altura de la base del exopodito (Vinogradov et al. 1996). Además, la proyección del margen distal de la base del pereiópodo 5 se extiende hasta la mitad del mero en $S$. damasi, mientras que en $S$. excisa la proyección no rebasa la base del mero (Zeidler, 1990).

Comentarios generales. Esta especie prefiere habitar aguas tropicales de la zona mesopelágica entre 200 y $500 \mathrm{~m}$ de profundidad (Thurston, 1976a; Vinogradov et al. 1996; Vinogradov, 1999). En el Pacífico este, $S$. excisa se registró al norte de México (Gasca, 2009b) y al sur de Perú
(Vinogradov, 1991), lo que representa a una amplitud en su distribución de aprox. $2600 \mathrm{~km}$ al sur y al norte del Pacífico americano.

Suborden SENTICAUDATA Lowry \& Myers, 2013

Familia CALLIOPIIDAE G. O. Sars, 1893

Género Stenopleura Stebbing, 1888 Stenopleura atlantica Stebbing, 1888 (Fig. 4C)

Diagnóstico. Cuerpo delgado. Ojos rojos reniformes. Gnatópodos 1-2 subquelados, con un lóbulo grande en el margen posterior del mero. Pereiópodos 3-7 largos y simples. Urópodos con sus márgenes laterales armados con espinas. Telson tan ancho como largo, el margen distal del telson con incisiones. Fuentes: Stebbing (1888), Vinogradov (1999), Violante-Huerta et al. (2020).

Material examinado. 16 individuos: 1 ind, est 3; 1 ind, est 4; 2 ind, est 6; 2 ind, est 8; 2 ind, est 10; 1 ind, est $11 ; 1$ ind, est $12 ; 1$ ind, est $13 ; 3$ ind, est $14 ; 1$ ind, est $15 ; 1$ ind est 21 .

Distribución global. Atlántico norte: Cuba, golfo de México, Irlanda, islas Canarias. Atlántico sur: Brasil, aguas oceánicas entre América y África, golfo de Guinea, Sudáfrica. Pacífico norte: Panamá. Pacífico sur: Nueva Zelanda. Océano Índico: mar Arábigo. Antártica: mar de Davis (Thurston, 1976b; Vinogradov, 1999; Bellan-Santini \& Costello, 2001; 
Lalana et al. 2005; De Broyer et al. 2007; Miloslavich et al. 2010; GBIF. org, 2020; OBIS, 2020; ViolanteHuerta et al. 2020).

Discusión taxonómica. Especie tipo del género monotípico Stenopleura (Horton et al. 2020). Stenopleura atlantica se caracteriza por poseer el margen distal del telson con incisiones, formando un margen tridentado, lo que la diferencia del género $\mathrm{Ca}$ lliopus, cuyo margen distal del telson es redondo (Barnard, 1964).

Comentarios generales. Stenopleura atlantica es común en aguas de la zona epipelágica de los océanos, especialmente del Atlántico (OBIS, 2020), sin embargo, la especie se ha observado en aguas de la zona mesopelágica hasta los $1000 \mathrm{~m}$ de profundidad en el Atlántico norte (Thurston, 1976b; Violante-Huerta et al. 2020). Stenopleura atlantica prefiere habitar aguas por encima de los $200 \mathrm{~m}$ de profundidad, debido a que, aparentemente, es la zona con las condiciones hidrológicas óptimas para su reproducción (Thurston, 1976b; Violante-Huerta et al. 2020).

\section{CONCLUSIÓN}

El registro de 13 especies de anfípodos pelágicos en las aguas oceánicas del Pacífico de Costa Rica contribuye al conocimiento de la diversidad de este importante grupo, para el país y el Pacifico este tropical. La lista actualizada de anfípodos pelágicos del Pacífico costarricense ahora es de 54 especies, lo que sugiere la necesidad de realizar más estudios al respecto, con miras a mejorar el conocimiento de la fauna marina de la región.

\section{AGRADECIMIENTOS}

Los autores agradecen a la Universidad de Costa Rica, la Universidad Nacional y al Parque Nacional Isla del Coco por su respaldo en la realización de la campaña oceanográfica UCR-UNA-COCO-VI y a Fabiola Jiménez por su apoyo durante el muestreo. El primer autor agradece el soporte financiero otorgado por la Universidad Nacional Autónoma de México / Posgrado en Ciencias del Mar y Limnología y al Consejo Nacional de Ciencia y Tecnología por la beca otorgada (CONACYT-862851) en México para llevar a cabo la estancia de investigación en el CIMAR de la Universidad de Costa Rica. Finalmente, los autores agradecen también a los revisores anónimos, por los valiosos comentarios que contribuyeron a mejorar este trabajo. 


\section{REFERENCIAS}

Barnard, J. L. (1964). Revision of some families, genera and species of Gammaridean Amphipoda. Crustaceana, 7(1), 49-74. https://doi. org/10.1163/156854064X00263

Barnard, J. L. (1972). A review of the family Synopiidae (= Tironidae), mainly distributed in the deep sea (Crustacea: Amphipoda). Smiths. Contr. Zool., 124, 1-94. https://doi.org/10.5479/ si.00810282.124

Barnard, J. L. \& Karaman, G. (1991). The Families and Genera of Marine Gammaridean Amphipoda (Except Marine Gammaroids). Rec. Austral. Mus., 13(2), 1-866. https://doi.org/10.3853 /j.0812-7387.13.1991.367

Barnard, J. L. \& Thomas, J. D. (1989). Four species of Synopiidae from the Caribbean region (Crustacea: Amphipoda). Proc. Biol. Soc. Wash., 102(2), 362-374.

Bellan-Santini, D. \& Costello, M. J. (2001). Amphipoda. En M. Costello (Ed.), European register of marine species: a check-list of the marine species in Europe and a bibliography of guides to their identification (pp. 295308). France: Collection Patrimoines Naturels.

Burridge, A. K., Tump, M., Vonk, R., Goetze, E. \& Peijnenburg, K. T. (2017). Diversity and distribution of hyperiid amphipods along a latitudinal transect in the Atlantic Ocean. Prog. Oceanogr., 158, 224-235. https://doi.org/10.1016/j. pocean.2016.10.001

De Broyer, C., Lowry, J. K., Jazdzewski, K. \& Robert, H. (2007). Catalogue of the Gammaridean and Corophiidean Amphipoda (Crustacea) of the Southern Ocean, with distribution and ecological data. En C. De Broyer (Ed.), Census of
Antarctic Marine Life: Synopsis of the Amphipoda of the Southern Ocean (pp. 1-325). Belgium: Bulletin de l'Institut royal des Sciences naturelles de Belgique.

Dick, R. I. (1967). A study of the Hyperiid Amphipoda in the waters off the east and south coasts of the Republic of South Africa in relation to prevailing hydrological conditions (unpublished doctoral thesis). University of Cape Town, South Africa.

Gasca, R. (2009a). Hyperiid Amphipods. In S. Wehrtmann \& J. Cortés (Eds.), Marine Biodiversity of Costa Rica. Central America (pp. 275-282). Germany: Springer. https://doi. org/10.1007/978-1-4020-8278-8_25

Gasca, R. (2009b). Hyperiid Amphipods (Crustacea: Peracarida) in Mexican Waters of the Pacific Ocean. Pac. Sci., 63(1), 8396. https://doi.org/10.2984/1534-6188 (2009)63[83:HACPIM]2.0.CO;2

Gasca, R. (2009c). Diversity of hyperiid amphipods (Crustacea: Peracarida) in the Western Caribbean Sea: news from the deep. Zool. Stud., 48(1), 63-70.

Gasca, R. \& Morales-Ramírez, A. (2012). Anfípodos hiperídeos (Crustacea: Peracarida) del Parque Nacional Isla del Coco, Costa Rica, Pacífico Tropical Oriental. Rev. Biol. Trop., 60(3), 223-233.

GBIF.org. (2020). GBIF Occurrence Download (https://doi.org/10.15468/39omei). https://www.gbif.org

Horton, T., Lowry, J., De Broyer, C., Bellan-Santini, D., Coleman, C. O., Corbari, L., ... \& Zeidler, W. (2020). World Amphipoda Database. Amphipoda. World Register of Marine Species (WoRMS). http://www.marinespecies. org/aphia.php? $\mathrm{p}=$ taxdetails\&id $=1135$

Hughes, L. E. (2009). Synopiidae. In J. K. Lowry \& A. A. Myers (Eds.), Benthic Amphipoda (Crustacea: Peracarida) 
of the Great Barrier Reef, Australia (pp. 880-891). New Zealand: Zootaxa, 2260. https://doi.org/10.11646/ zootaxa.2260.1.50

Lalana, R., Ortiz, M. \& Varela, C. (2005). Primera adición a la lista de los crustáceos no decápodos de Cuba. Rev. Biol., 19, 50-56.

Ledoyer, M. (1986). Crustacés amphipodes gammariens: familles des Haustoriidae à Vitjazianidae. Faune Madagascar, 59, 599-1112.

Miloslavich, P., Díaz, J. M., Klein, E., Alvarado, J. J., Díaz, C., Gobin, J., ... \& Ortiz, M. (2010). Marine biodiversity in the Caribbean: regional estimates and distribution patterns. PLoS ONE, 5, e11916. https://doi.org/10.1371/journal.pone.0011916

OBIS. (2020). Ocean Biogeographic Information System (OBIS). https://obis.org/

Salmán-Palacios, C. (1985). Sistemática y distribución de los hipéridos pelágicos (Crustacea: Amphipoda) del domo de Costa Rica (tesis de licenciatura no publicada). Universidad Nacional Autónoma de México, México.

Senna, A. R., Andrade, E. F., Guedes, U. N. \& Pereira-Filho, G. H. (2020). Redescription and new records of Synopia ultramarina Dana (Amphipoda: Synopiidae) from off the northeastern Brazil, with comments on its morphological variations. Iheringia, Sér. Zool., 110, 1-11. https://doi. org/10.1590/1678-4766e2020017

Shih, C. T. \& Hendrycks, E. A. (2003). A new species and new records of the genus Vibilia Milne Edwards, 1830 (Amphipoda: Hyperiidea: Vibiliidae) occurring in the eastern Pacific Ocean. J. Nat. Hist., 37, 253-296. https://doi. org/10.1080/713834685

Shoemaker, C. R. (1945). The Amphipoda of the Bermuda Oceanographic
Expeditions, 1929-1931. Zoologica, 30(4), 185-266.

Shulenberger, E. (1978). Vertical distribution, diurnal migration, and sampling problems of hyperiid amphipods in the North Pacific central gyre. DeepSea Res., 25, 605-623. https://doi. org/10.1016/0146-6291(78)90616-1

Stebbing, T. R. R. (1888). Report on the Amphipoda collected by H. M. S. Challenger during the years 1873-1876. Report on the scientific results of the Voyage of H. M. S. Challenger during the years 1873-76. Zool., 29, 1-1737.

Thurston, M. H. (1976a). The vertical distribution and diurnal migration of the Crustacea Amphipoda collected during the SOND Cruise, 1965 II. The Hyperiidea and general discussion. J. Mar. Biol. Assoc. U. K., 56(2), 383-470. https:// doi.org/10.1017/S0025315400018981

Thurston, M. H. (1976b). The vertical distribution and diurnal migration of the Crustacea Amphipoda collected during the Sond Cruise, 1965: I. The Gammaridea. J. Mar. Biol. Assoc. U. K., 56(2), 359-382. $\quad$ https://doi.org/10.1017/ S002531540001897X

Valencia, B., Lavaniegos, B., Giraldo, A. \& Rodríguez-Rubio, E. (2013). Temporal and spatial variation of hyperiid amphipod assemblages in response to hydrographic processes in the Panama Bight, eastern tropical Pacific. DeepSea Res. Part I, 73, 46-61. https://doi. org/10.1016/j.dsr.2012.11.009

Vinogradov, G. M. (1991). Hyperiid amphipods in the eastern part of the South $\mathrm{Pa}$ cific gyre. Mar. Biol., 109(2), 259-265. https://doi.org/10.1007/BF01319394

Vinogradov, G. (1999). Amphipoda. In D. Boltovskoy (Ed.), South Atlantic Zooplankton (pp. 1141-1240). Netherlands: Backhuys Publishers. 
Vinogradov, M. E., Volkov, A. F. \& Semenova, T. N. (1996). Hyperiid Amphipods (Amphipoda, Hyperiidea) of the World Oceans. EE. UU.: Science Publ. Inc., Lebanon.

Violante-Huerta, M. (2019). Estructura de la comunidad de anfipodos (Crustacea: Peracarida) planctónicos del sur del Golfo de México (tesis de maestría no publicada). Universidad Nacional Autónoma de México, México.

Violante-Huerta, M., Sanvicente-Añorve, L., Marrón-Becerra, A., Lemus-Santana E. \& Flores-Coto, C. (2020). First records of non-hyperiid planktonic amphipods (Amphilochidea and Senticaudata) from deep waters of the southern Gulf of Mexico, with an identification key. Rev. Mex. Biodivers., 91, e912975.

Zeidler, W. (1990). Pelagic amphipods, Infraorder Physosomata (Crustacea: Amphipoda: Hyperiidea) from the CSK International Zooplankton Collection (Western North Pacific), with the description of four new species of Scina. Publ. Seto Mar. Biol. Lab., 34(4/6), 167200. https://doi.org/10.5134/176167

Zeidler, W. (2003). A review of the hyperiidean amphipod superfamily Vibilioidea Bowman and Gruner, 1973 (Crustacea:
Amphipoda: Hyperiidea). Zootaxa, 280, 1-104. https://doi.org/10.11646/ zootaxa.280.1.1

Zeidler, W. (2004). A review of the families and genera of the hyperiidean amphipod superfamily Pronimoidea Bowman \& Gruner, 1973 (Crustacea: Amphipoda: Hyperiidae). Zootaxa, 567, 1-66. https://doi.org/10.11646/ zootaxa.567.1.1

Zeidler, W. (2016). A review of the families and genera of the superfamily Platysceloidea Bowman \& Gruner, 1973 (Crustacea: Amphipoda: Hyperiidea), together with keys to the families, genera and species. Zootaxa, 4192, 1-136. https://doi.org/10.11646/ zootaxa.4192.1.1

Zhang, W., Lin, Y., He, C., Cao, W., Huang, J., Zheng, L., Yang, W. \& Wang, Y. (2014). Hyperiid amphipod communities and the seasonal distribution of water masses in eastern Beibu Gulf, South China Sea. Aquat. Biol., 20(3), 209217. https://doi.org/10.3354/ab00556 
